\title{
Skin Ulceration, CTCAE
}

National Cancer Institute

\section{Source}

National Cancer Institute. Skin Ulceration, CT CAE. NCI Thesaurus. Code C143839.

A disorder characterized by a circumscribed, erosive lesion on the skin. 